\title{
Spontaneous recovery in cross-motivational transfer (counterconditioning)
}

\author{
MARK E. BOUTON and CHARLES A. PECK \\ University of Vermont, Burlington, Vermont
}

\begin{abstract}
In two experiments with rat subjects, we examined the effects of a retention interval on performance in two conditioning paradigms in which a conditioned stimulus (CS) was associated with different unconditioned stimuli (USs) in successive phases of the experiment. Experiment 1 was designed to examine aversive-appetitive transfer, in which the CS is associated with shock and then food; Experiment 2 was designed to examine appetitive-aversive transfer, in which the CS is associated with food and then shock. Aversive and appetitive conditioned responses (freezing and head-jerk responding, respectively) were scored from videotape. In both experiments, a 28 day retention interval following the end of Phase 2 caused a recovery of the Phase 1 response and a resuppression of the Phase 2 response. The results suggest that the original association is not destroyed when the CS is associated with a new US in Phase 2. They also suggest that both retroactive and proactive interference effects may result from interference with performance output rather than a disruption or loss of what is learned during or stored from the target phase.
\end{abstract}

The present article is concerned with two conditioning paradigms in which a conditioned stimulus (CS) is paired with different unconditioned stimuli (USs) in successive phases of the experiment. In aversive-to-appetitive transfer, the CS is associated with an aversive US (e.g., shock) in an initial phase and then an appetitive US (e.g., food or water) in a second phase. In appetitive-to-aversive transfer, the CS is first paired with an appetitive US and then an aversive US. In either case, the CS comes to evoke responding appropriate to the second US by the end of the second phase (see, e.g., Bromage \& Scavio, 1978; DeVito \& Fowler, 1982; Dickinson, 1976; Jackson, 1974; Konorski \& Szwejkowska, 1956; Krank, 1985; Peck \& Bouton, 1990; Scavio, 1974; Scavio \& Gormezano, 1980). In aversive-to-appetitive transfer, Phase 1 aversive conditioning also retards the CS's acquisition of Phase 2 appetitive responding (Bromage \& Scavio, 1978; Krank, 1985; Peck \& Bouton, 1990; Scavio, 1974). The results are more mixed in appetitive-to-aversive transfer, where initial appetitive conditioning has retarded (Dickinson, 1976; Konorski \& Szwejkowska, 1956), facilitated (DeVito \& Fowler, 1982; Scavio \& Gormezano, 1980), or had no effect on (Jackson, 1974; Peck \& Bouton, 1990) aversive conditioning in the second phase.

Research on these "cross-motivational transfer" paradigms has been guided by the view that the aversive and appetitive motivational systems inhibit one another reciprocally (see, e.g., Dickinson \& Dearing, 1979; Konorski, 1967; Rescorla \& Solomon, 1967). In associa-

This research was supported by Grant BNS 89-08535 from the $\mathrm{Na}$ tional Science Foundation. We thank D. C. Brooks for his comments on the manuscript. Send correspondence to M. E. Bouton, Department of Psychology, University of Vermont, Burlington, VT 05405. tive terms, a CS associated with a US of one type is seen as equivalent to a conditioned inhibitor within the other motivational system (see, e.g., Dickinson \& Dearing, 1979; Krank, 1985; see also Daly \& Daly, 1982, 1987, for an extension of this analysis). In principle, this view allows one to put cross-motivational transfer into the terms of conditioning models that otherwise cannot deal with conditioning phenomena involving more than one US. The best-known of these models is probably the RescorlaWagner model (Rescorla \& Wagner, 1972; see Krank, 1985). This model assumes that the result of conditioning is a change in a single variable called associative strength. Conditioned excitors and inhibitors are assumed to have positive and negative values of associative strength, respectively. In aversive-to-appetitive transfer, for example, the CS would initially acquire "negative" appetitive strength, but in the second phase it would eventually acquire "positive" appetitive strength.

The implications of this perspective are straightforward. Because the CS begins Phase 2 with a negative value, it is slower than a novel CS to acquire a positive value during Phase 2. In addition, because the product of Phase 2 is a change in the single associative strength variable, conversion of the negative CS into a positive one will abolish its negative strength. The application of the RescorlaWagner model makes explicit what might be implicit in more casual descriptions from the reciprocal-inhibition framework: Acquisition in Phase 2 is assumed to be slow because the subject is slow to learn the new CS-US association, and acquisition of the second association may destroy the aversive association that was learned in Phase 1. The same assumptions are present in recent applications of the Rescorla-Wagner model, also known as the delta rule or least-mean-squares rule, to human learn- 
ing tasks in which different stimuli or features are associated with more than one outcome or category (e.g., Gluck \& Bower, 1988; see also Shanks, 1991).

A different perspective on cross-motivational transfer is also possible. The paradigm is but one of a set of "interference paradigms" in Pavlovian learning in which a CS is paired with different outcomes in different phases of the experiment (Bouton, 1991). Bouton (1991) and Spear (1981) have suggested that such paradigms can be viewed as memory retrieval problems in which information from both phases is stored and ready to be retrieved by the corresponding retrieval cues. What determines performance is not the current value of a single associative strength variable, but which of the two available memories is retrieved. For example, after extinction (one of the interference paradigms), either conditioning or extinction performance can be cued by manipulation of background contextual cues. If conditioning and extinction are conducted in different contexts, a return to the conditioning context after extinction can "renew" the Phase 1 conditioned response (CR) (see, e.g., Bouton \& Bolles, 1979; Bouton \& King, 1983; Bouton \& Peck, 1989). Such a result, among others, suggests that the Phase 1 association is not destroyed during Phase 2 (see Bouton, 1991, for a review).

Peck and Bouton (1990) reported similar renewal effects in both aversive-to-appetitive and appetitive-toaversive transfer. When the CS was paired with one US in one context and then the other US in another context, a return to the first context (1) renewed the Phase 1 CR and (2) resuppressed the Phase $2 \mathrm{CR}$. The results suggested that the Phase 1 association had not been destroyed by Phase 2 . In addition, since the Phase 2 association clearly had been learned by the end of Phase 2 , the resuppression of the Phase 2 CR during the test suggested that proactive interference can come about through interference with Phase 2 performance rather than Phase 2 learning.

Bouton (1991) noted that context manipulations have important effects in virtually all of the Pavlovian interference paradigms. In addition, the manipulation of time may also have compatible effects. For example, if time is allowed to elapse after extinction, the CR often recovers (see, e.g., Pavlov, 1927). Like the renewal effect, "spontaneous recovery" suggests that Phase 2 does not completely destroy what was learned in Phase 1 . The present experiments were designed to extend our previous results with physical context (Peck \& Bouton, 1990) by asking whether time has analogous effects in cross-motivational transfer. To the best of our knowledge, the question has not been addressed before. Spontaneous recovery in crossmotivational transfer is not directly anticipated by the reciprocal inhibition view, or by its adaptation to the Rescorla-Wagner model, because they imply the unlearning of the Phase 1 association during Phase 2. Conditioning theory has rarely addressed the possible effects of retention interval on performance in Pavlovian learning.

\section{EXPERIMENT 1}

In the first experiment, we examined spontaneous recovery in aversive-to-appetitive transfer. Two groups of rats received initial pairings of a tone CS with a footshock US. In the second phase, the tone was then paired with a food-pellet US. Following the conclusion of appetitive conditioning, one group was tested at a 1-day retention interval and the other group was tested after 28 days. Two control groups received similar appetitive conditioning and then tests at the 1- and 28-day intervals, but they had no initial aversive conditioning and thus no basis for spontaneous recovery. We used Peck and Bouton's (1990) method. The rats' response to the CS was videotaped and scored for the occurrence of both aversive and appetitive CRs. The aversive CR consisted of freezing, a natural defensive response of the rat to CSs associated with footshock (see, e.g., Bolles \& Collier, 1976; Bouton \& Bolles, 1980; Fanselow \& Bolles, 1979; Holland, 1979). The appetitive CR was head-jerk responding, a response that the rat makes to auditory CSs that have been associated with food (see, e.g., Bouton \& Peck, 1989; Holland, 1977, 1979). If aversive-to-appetitive transfer is like extinction, freezing might recover over time. We were also interested in whether there would be a complementary effect on head-jerk responding at the longer retention interval.

\section{Method}

\section{Subjects}

The subjects were 32 female Wistar rats bred at the University of Vermont. They were 170 days old at the start of the experiment. The rats were housed individually in standard stainless steel cages in a room maintained on a 16:8-h light:dark cycle; the experiment was conducted on consecutive days during the light portion of the cycle. All rats were food deprived and maintained at $80 \%$ of their initial body weights except where noted.

\section{Apparatus}

Four sets of four conditioning chambers located in separate rooms were used in various parts of the experiment. The sets have been used as separate "contexts" in other work conducted in this laboratory, although they were not used in that capacity here. In the first set, each box measured $26 \times 25 \times 19 \mathrm{~cm}$. The back and two side walls were constructed of aluminum, and the front wall and ceiling were made of clear acrylic plastic. The floor consisted of tubular steel bars $16 \mathrm{~mm}$ in diameter, spaced $3.2 \mathrm{~cm}$ center to center. These bars were parallel to the front wall. A food cup protruded from the right wall and was centered $1 \mathrm{~cm}$ above the floor and $1 \mathrm{~cm}$ to the left of a nonfunctional $2.5 \times 2.5 \mathrm{~cm}$ lever. The rats were placed in the chamber through a door in the front wall. Illumination was provided by a 7.5-W red incandescent bulb mounted on the ceiling $28 \mathrm{~cm}$ above the floor. A dish containing approximately $10 \mathrm{ml}$ of Heinz white vinegar was positioned behind the right wall (outside the chamber) to provide a distinctive scent cue.

The second set of four boxes measured $24 \times 22 \times 18 \mathrm{~cm}$. The two side walls were aluminum; the front and back walls and ceiling were clear acrylic plastic with vertical black stripes, which were $2 \mathrm{~cm}$ wide and were spaced $2.5 \mathrm{~cm}$ apart. The floor consisted of stainless steel bars, which were $3 \mathrm{~mm}$ in diameter and were spaced $1.5 \mathrm{~cm}$ center to center and mounted parallel to the sidewalls. A food cup was recessed in the right wall $1 \mathrm{~cm}$ above the floor and 
$3.5 \mathrm{~cm}$ (from its center) from the front wall. A $1-\mathrm{cm}$-wide lever (nonfunctional) protruded $4 \mathrm{~cm}$ into the box and was positioned in the center of the right wall $5 \mathrm{~cm}$ above the floor and $6 \mathrm{~cm}$ to the left of the food cup. The rats were placed in the chamber through the ceiling of the box. Illumination was provided by a $7.5-\mathrm{W}$ red incandescent bulb mounted on the ceiling $27 \mathrm{~cm}$ above the floor, where a speaker, the source on the CS, was also mounted. Approximately $1 \mathrm{~g}$ of Vicks Vaporub was smeared daily on the top of the housing surrounding the recessed food cup (outside of the box) to provide a distinctive scent cue.

Two other sets of four boxes were used during magazine training. All these boxes were made of clear acrylic plastic and measured $23 \times 13 \times 11 \mathrm{~cm}$. The walls were covered on the outside with black construction paper, except for the front wall (and ceiling), which was transparent. A small $(1 \times 1 \mathrm{~cm})$ food cup protruded from the back wall, $4.5 \mathrm{~cm}$ from the floor and $4.5 \mathrm{~cm}$ from the right wall. Illumination was provided by a $7.5-\mathrm{W}$ red incandescent bulb mounted on the ceiling of the sound-attenuation chamber, $25 \mathrm{~cm}$ above the floor, next to a speaker, the source of the CS. The subjects were placed in the boxes through the ceiling.

One set of boxes had floors consisting of 3-mm bars mounted parallel to the right $(11-\mathrm{cm})$ wall. The bars were staggered so that the odd-numbered bars were mounted $6 \mathrm{~mm}$ above the evennumbered bars; the distance between consecutive bars was $1.6 \mathrm{~cm}$. The three black (covered) walls were lined with horizontal white stripes, which were $1 \mathrm{~cm}$ wide and were spaced $1 \mathrm{~cm}$ apart. Within each sound-attenuation chamber, a dish containing $10 \mathrm{ml}$ of $4 \%$ McCormick coconut extract solution provided a distinctive scent cue. The second set of boxes had floors consisting of 3-mm bars spaced $1.8 \mathrm{~cm}$ apart and mounted diagonally with respect to the chamber walls. The three covered walls remained black. A dish of $2 \%$ McCormick anise extract solution was positioned in the sound-attenuation chamber to provide the distinctive scent cue.

The sound-attenuation chambers housing any set of boxes were positioned together in a $2 \times 2$ arrangement. Clear acrylic plastic windows (double-paned) in the chamber fronts permitted a view of the boxes within. A Panasonic low-light camera (Model WV1460), fitted with an 8-mm wide-angle lens, was mounted $3.1 \mathrm{~m}$ away from the sound-attenuation chambers so that it could record activity in all four chambers at one time. Video signals from each room were recorded in a separate room by Panasonic video-cassette recorders (Model AG-2200) controlled by an AT\&T 6300 microcomputer. All recording and programming equipment was located in this other room.

The CS was always a $30-\mathrm{sec}$ presentation of a $3000-\mathrm{Hz}$ tone ( $80 \mathrm{~dB}$ re $20 \mu \mathrm{N} / \mathrm{m}^{2}$ [A]) provided by a single generator wired to identical speakers in each chamber. The background noise level was $65 \mathrm{~dB}$. The appetitive US consisted of two 45-mg Noyes food pellets (Formula A) delivered $0.2 \mathrm{sec}$ apart. The aversive US was a 0.6-mA 0.5 -sec scrambled footshock provided by Grason-Stadler shock sources.

\section{Procedure}

Pretraining. Prior to the start of aversive conditioning, magazine training was conducted in either an anise- or a coconut-scented box. On Day 1, all rats were exposed to the magazine-training boxes in a 30-min session with foodcups baited with several pellets. On Day 2, they were exposed to either a vinegar- or a Vick's-scented box in a single 30-min session with no food present. On Day 3, the rats were returned to the magazine-training boxes and, in a 20 min session, were hand shaped to eat food pellets when they were delivered to the food cup. At the start of the session the cup was baited with two pellets; rats received an average of eight appetitive USs during the session. All subsequent sessions were conducted in the box preexposed on Day 2.

Aversive conditioning. On the day following pretraining, the rats were randomly divided into two conditions, interference and noninterference $(n=16)$. On each of the next 3 days, the rats in the interference condition received a 90 -min session containing six tone-shock pairings in which CS offset coincided with shock onset. The intertrial intervals (ITIs) averaged $12.4 \mathrm{~min}$; no events $\propto c$ curred until at least 5 min into the session. The rats in the noninterference condition received similar sessions without tones or shocks. On each of the 4 days that followed the final aversive conditioning session, every rat received a daily 50 -min session of box exposure designed to extinguish any fear conditioned to the apparatus. The interference rats received no tones or shocks during any of these sessions. The noninterference rats, however, received two tones alone in the last exposure session in order to reduce any unconditional responding to the tone prior to beginning Phase 2 . No other stimuli were presented on this or any of the preceding box exposure days.

Appetitive conditioning. The rats from both conditions then received five daily 50 -min sessions of appetitive conditioning. In each session, there were four pairings of the tone and food-pellet US; once again, CS offset coincided with US onset. The ITI was variable, with an average of $12.7 \mathrm{~min}$; no events occurred in the first 5 min of any session.

Retention test. On the day following the last appetitive conditioning day, the rats within each condition were divided into two new subgroups that were matched on freeze and head-jerk responding during appetitive conditioning. One group from each interference condition (Groups II and NI) was then tested for responding to the tone 1 day after the completion of appetitive conditioning. The remaining groups ( 128 and $\mathrm{N} 28$ ) were tested 28 days later. The 28-day groups were returned to ad-lib feeding for the first 18 days of the retention interval and were then redeprived to $80 \%$ of their current free-feeding weights. For all groups, testing consisted of six presentations of the tone alone (mean ITI $=7 \mathrm{~min}$ ) in a single 50 -min session. The first tone was presented during the 5 th $\mathrm{min}$ of the session. No USs of any kind were delivered during the test session.

Observation procedure. Throughout the experiment, behavior was scored by an observer who viewed the videotaped record after each session. Using a time-sampling technique (see, e.g. Bouton \& Peck, 1989; Holland, 1977; Peck \& Bouton, 1990), the observer scored each rat five times during the 30 -sec interval preceding the $\mathrm{CS}$ (the pre-CS period) and five times during the CS. Observations were driven by a small light (recorded on tape) that flashed at 1.5 $\mathrm{sec}$ intervals beginning $0.75 \mathrm{sec}$ into the pre-CS period. Four rats were scored at a time; observations shifted between rats in a regular sequence. The observer was free to rescore any trial as needed to ensure an accurate account of every trial. The observer scored two behaviors: head jerk and freeze. Head jerk, the measure of appetitive excitation to the tone, was defined as short rapid head movements independent of other movements of the body (cf. Bouton \& Peck, 1989; Holland, 1977, 1979; Peck \& Bouton, 1990). Freezing, the index of aversive conditioning, was defined as complete body immobility (see, e.g., Bouton \& Bolles, 1979; Peck \& Bouton, 1990).

The reliability of the observation procedure was evaluated during the focal test session. The original observer scored the entire session twice, once at the end of the experiment, and again several months later while blind to group assignment. When the two sets of data were compared, agreement did not vary across behaviors and averaged $94.5 \%$ in the pre-CS period and $94.3 \%$ in the CS period. The two data sets were also subjected to identical statistical analyses; the results supported the same conclusions. Finally, $25 \%$ of the video record from each group was independently scored by a second observer who was blind to the experimental treatments. Agreement with the primary observer averaged $93 \%$ in the pre-CS period and $87.5 \%$ in the CS period. Once again, it was consistent over both head jerk and freezing.

Data analysis. Observations scored as head jerk and freezing were converted to percentage behavior scores by dividing the number of samples of each type by the total number of samples. These were 
then analyzed with separate analyses of variance (ANOVAs); preCS responding was also routinely analyzed with ANOVAs identical to the ones used on CS responding. Planned comparisons were conducted according to the standard methods discussed by Howell (1987, p. 332ff.). The rejection criterion was set at $p<.05$.

\section{Results}

The data from Phase 2 (appetitive conditioning) and retention testing are shown in the left and right portions of Figure 1. The results confirmed that, while head-jerk responding came to replace freezing when the tone was paired with the food-pellet US during Phase 2 , head jerk then became suppressed and was replaced by freezing at the 28-day retention interval.

\section{Appetitive Conditioning (Phase 2)}

Freezing. Freezing to the CS during each trial of Phase 2 is shown at the left in the lower panel of Figure 1. Statistical analyses confirmed that the tone-shock pairings received by the interference groups (I1 and I28) during Phase 1 caused initial freezing that declined over Phase 2. An interference $\times$ retention interval $\times$ trial ANOVA revealed significant main effects of interference $[F(1,28)=32.02]$ and trial $[F(19,532)=12.79]$, as well as a significant interference $\times$ trial interaction $[F(19,532)=4.74]$. No main effects or interaction involving retention interval were reliable $(F \mathrm{~s}<1.45)$. The rats in the interference condition (Groups 11 and I28) ini-
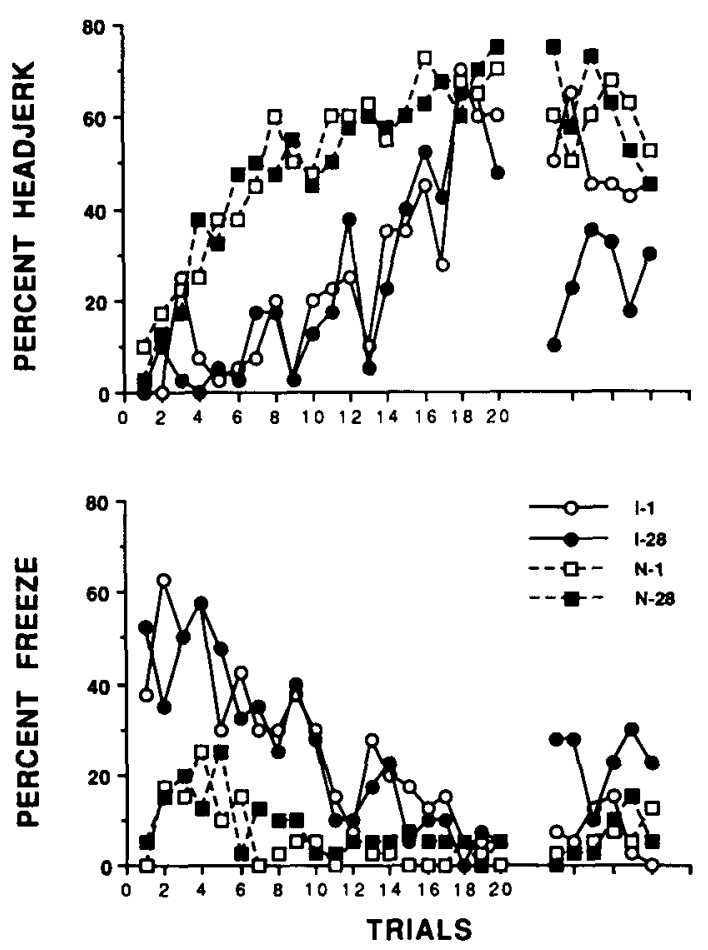

Figure 1. Results of Phase 2 (left) and retention testing (right) during Experiment 1. The top panel shows head-jerk responding, the Phase 2 conditioned response (CR). The bottom panel shows freezing, the Phase $1 \mathrm{CR}$. tially froze more than the rats in the noninterference condition (Groups N1 and N28). Planned comparisons isolating Trial 1 confirmed that Groups I1 and I28 froze more than Groups N1 and N28 [Fs $(1,310)>11.33]$.

Freezing during the pre-CS periods of the phase averaged $26.0 \%, 30.0 \%, 21.0 \%$ and $19.8 \%$ for Groups I1, $\mathrm{I} 28, \mathrm{~N} 1$, and $\mathrm{N} 28$, respectively. An interference $\times$ retention interval $x$ trial ANOVA on pre-CS freezing revealed no effects of interference or retention interval and no interference $\times$ retention interval interaction $[F \mathrm{~s}(1,28)<$ $2.60]$. Both the trial effect $[F(19,532)=5.28]$ and the interference $\times$ trial interaction $[F(19,532)=1.79]$ were significant. The interference groups began the phase with some pre-CS freezing, but it declined over the phase. There were no significant interactions involving retention interval (all $F$ s $<1$ ).

Head-jerk responding. The left portion of Figure 1's upper panel shows the acquisition of head-jerk responding to the tone during Phase 2. The figure suggests that the interference groups (I1 and I28) were slower to acquire head jerking than were the noninterference groups (N1 and N28). This impression was confirmed by an in'terference $\times$ retention interval $\times$ trial ANOVA on responding during the $\mathrm{CS}$ period. The analysis revealed significant main effects of interference $[F(1,28)=95.37]$ and trial $[F(19,532)=25.88]$, as well as an interference $x$ trial interaction $[F(19,532)=3.47]$. No main effects or interactions involving retention interval were reliable $(F \mathrm{~s}<1)$. There was very little head-jerk responding during the pre-CS periods of the phase; Groups I1, I28, $\mathrm{N} 1$, and $\mathrm{N} 28$ averaged $0.8 \%, 0.8 \%, 0.8 \%$, and $1.4 \%$, respectively.

\section{Retention Test}

Freezing. The right-hand portion of the figure suggests that freezing recovered over the 28-day retention interval in the interference group (I28). An interference $X$ retention interval $\times$ trial ANOVA on the test data suggested that this was true: There were significant effects of interference $[F(1,28)=9.43]$ and retention interval $[F(1,28)=7.08]$ as well as an interference $\times$ retention interval interaction $[F(1,28)=7.08]$. No effects involving the trials factor were significant $[F \mathrm{~s}(5,140)<1.79]$. Planned comparisons confirmed that Groups I1 and I28 differed $[F(1,28)=14.16]$, whereas Groups $\mathrm{N} 1$ and $\mathrm{N} 28$ did not $[F(1,28)<1]$.

Interpretation of the data on the later test trials was somewhat complicated by the fact that freezing during the pre-CS period tended to increase over testing: An analysis of pre-CS freezing revealed a trial effect $[F(5,140)=$ 5.59], but no other main effects or interactions (all $F \mathrm{~s}<$ 1.69). We therefore conducted a separate analysis of the CS-freezing on Trial 1, where there was relatively little freezing during the pre-CS period. The Trial 1 ANOVA revealed a significant main effect of interference $[F(1,28)=10.11]$ and a significant interference $\times$ retention interval interaction $[F(1,28)=4.84]$. The retention interval main effect was not reliable $[F(1,28)=2.93, p=$ 
.098]. Planned comparisons revealed that Group I 28 froze more than Group I1 $[F(1,28)=7.65]$, while Groups N1 and $\mathrm{N} 28$ did not differ $[F(1,28)<1]$. An identical analysis of pre-CS freezing revealed no corresponding differences among the groups $[F \mathrm{~s}(1,28)<2.04]$. The results strongly suggest that $C S$ freezing had recovered partially in Group 128 at the 28 -day retention interval.

Head-jerk responding. The upper panel of Figure 1 also suggests that a suppression of head-jerk responding occurred over time. An interference $\times$ retention interval $x$ trials ANOVA on head-jerk responding during the CS revealed significant main effects of interference $[F(1,28)=$ $32.16]$ and retention interval $[F(1,28)=7.33]$, and an interference $\times$ retention interval interaction $[F(1,28)=$ 10.36]. No effects involving the trials factor were significant $[F s(5,140)<1.83]$. Planned comparisons revealed that Group 128 head-jerked less than Group $\operatorname{Il}[F(1,28)=$ 17.54], while there was no difference between the corresponding noninterference groups $[F(1,28)<1]$. These conclusions were consistent with a similar analysis isolating Trial 1, which revealed a significant effect of interference $[F(1,28)=36.62]$ and an interference $\times$ retention interval interaction $[F(1,28)=19.69]$. The retention interval effect was marginally significant $[F(1,28)=4.69$, $p=.053$ l. Individual comparisons again revealed less head jerk in Group I 28 than in Group I1 $[F(1,28)=20.83]$ but no corresponding difference between Groups N28 and $\mathrm{N} 1[F(1,28)=2.93]$. These data suggest that head-jerk responding declined in the interference condition over the retention interval used in this experiment.

Pre-CS head-jerk responding averaged $0.0 \%, 0.4 \%$, $0.4 \%$, and $2.9 \%$ for Groups $\mathrm{I} 1, \mathrm{I} 28, \mathrm{~N} 1$, and $\mathrm{N} 28$, respectively, during the test. ANOVAs on the first trial and on the entire test revealed no significant main effects or interactions $(F \mathrm{~S}<2.93)$.

\section{Discussion}

During Phase 2, prior aversive conditioning interfered proactively with the acquisition of appetitive head-jerk responding; ultimately, appetitive conditioning came to interfere retroactively with aversive freezing. Both results are consistent with the literature on aversive-to-appetitive transfer (Bromage \& Scavio, 1978; Krank, 1985; Peck \& Bouton, 1990; Scavio, 1974). The new finding, however, is that these effects were modified by the passage of time. At the 28-day retention interval, freezing had partially recovered, while head-jerk responding had become resuppressed. Neither effect was evident in noninterference control groups that had received CS-food pairings without prior aversive conditioning. Overall, the data are consistent with the view that Phase 2 appetitive conditioning did not abolish what had been learned during Phase 1 aversive conditioning. In addition, aversive conditioning's proactive interference with appetitive head-jerk responding can evidently occur even after the CS-food association had been clearly learned during Phase 2 . Retroac- tive interference does not require the unlearning of Phase 1, and similarly, proactive interference does not require a failure of learning in Phase 2.

\section{EXPERIMENT 2}

In the second experiment, we used a similar design to ask whether appetitive-to-aversive transfer performance would be similarly affected by retention interval. The recovery effects in Experiment 1 could depend on an asymmetry between aversive and appetitive conditioning; the recovery of fear behavior and the resuppression of appetitive behavior might depend on fear taking priority over appetitive conditioning. This view would not predict a recovery of the Phase $1 \mathrm{CR}$ and resuppression of the Phase $2 \mathrm{CR}$ in appetitive-to-aversive transfer.

\section{Subjects and Apparatus \\ Method \\ The subjects were 32 female Wistar rats from the same stock as those in Experiment 1. They were approximately 140 days old at the start of the experiment and were housed and maintained as in Experiment 1. The apparatus was the same as before, except that only the anise- and coconut-scented sets of boxes were used in Ex- periment 2.}

\section{Procedure}

The animals first received pretraining following the procedure used in the previous experiment. Thereafter, all sessions were $50 \mathrm{~min}$ in duration and were conducted in the light portion of the day:night cycle. They also occurred on consecutive days, except as noted below.

Appetitive conditioning. On the day following the conclusion of pretraining, the rats were randomly divided into interference and noninterference conditions $(n=16)$. On each of the next 3 days, the rats in the interference condition received a session containing 12 tone-food pairings. The ITIs averaged $3.2 \mathrm{~min}$; no events were scheduled in the first $5 \mathrm{~min}$ of a session. The rats in the noninterference condition received equal apparatus exposure with no tones or food pellets. On the day immediately following the last appetitive conditioning day, all rats next received one 50 -min session of box exposure. The rats in the interference condition received no Pavlovian events, but the noninterference rats received two presentations of the tone alone to reduce any unconditioned responding prior to the beginning of the next phase (as in Experiment 1).

Aversive conditioning. Aversive conditioning consisted of four sessions that began on the next day. Each session contained a single tone-shock pairing in which tone onset occurred in the 5 th min of the session.

Retention test. The rats from each condition were then divided into final subgroups in a manner that matched them on freezing and head-jerk responding during aversive conditioning. Test sessions were then conducted at 1 - and 28-day retention intervals; treatment during the longer interval was the same as in Experiment 1. The test procedure (six nonreinforced trials in a 50 -min session) was also the same as in the preceding experiment.

Observation. Observational data were collected and evaluated as in Experiment 1. Agreement between the primary observer's first and second (blind) scoring of the entire test session did not vary according to behavior and averaged $94.3 \%$ in the pre-CS period and $86.7 \%$ in the CS period. Identical statistical analyses of the two data sets again supported the same conclusions. A random $25 \%$ 
of the trials of each group was again scored by a second blind observer; interobserver agreement was once again consistent and averaged $89.8 \%$ in the pre-CS period and $87.9 \%$ in the CS period.

\section{Results}

The main results of the experiment are presented in Figure 2, which shows freezing (top) and head-jerk responding (bottom) during Phase 2 aversive conditioning and during retention testing (right). Although freezing came to replace head-jerk responding during Phase 2, head jerk appeared to be recovered and freezing suppressed at the 28-day retention interval.

\section{Aversive Conditioning (Phase 2)}

Head-jerk responding. Head-jerk responding to the CS was evident in the interference groups at the outset of aversive conditioning, but it declined over the tone-shock pairings given during the phase. An interference $X$ retention interval $X$ trial ANOVA on CS responding revealed significant effects of interference $[F(1,28)=48.11]$ and trial $[F(3,84)=31.59]$, and an interference $\times$ trial interaction $[F(3,84)=16.75]$. No effects involving retention interval were significant $(F s<1)$. Planned comparisons isolating Trial 1 confirmed that Groups I1 and I28 each headjerked more than either Group N1 or Group N28 $[F \mathrm{~s}(1,95)>22.77]$. The Trial 1 differences, of course, merely confirm that tone-food pairings during Phase 1 were necessary to create head-jerk responding.
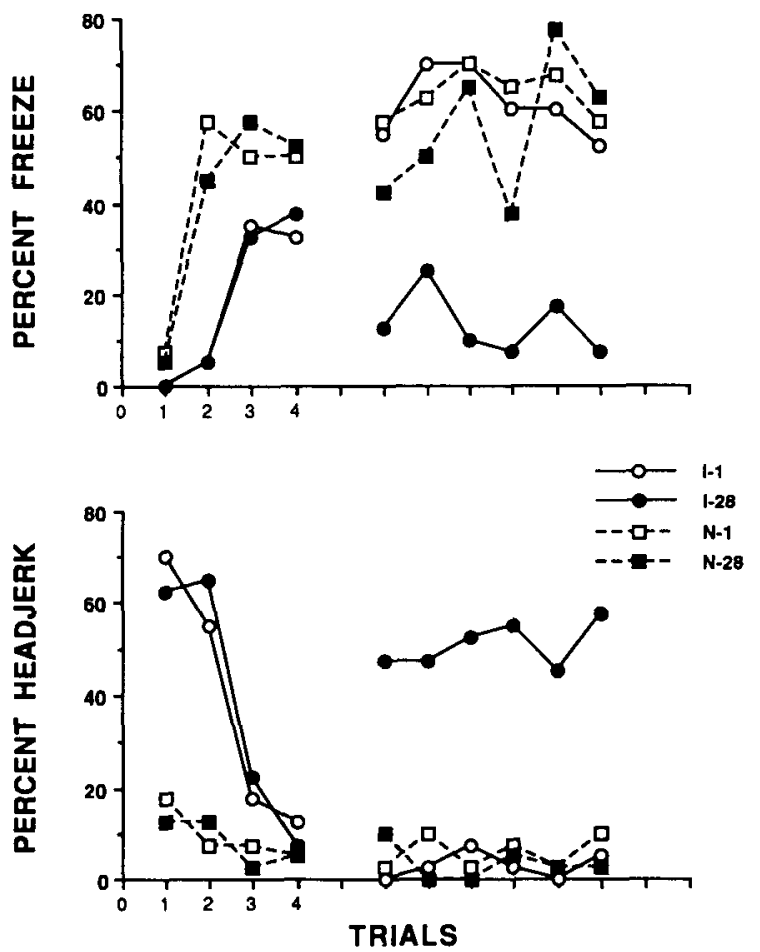

Figure 2. Results of Phase 2 (left) and retention testing (right) during Experiment 2. The top panel shows freezing, the Phase 2 conditioned response (CR). The bottom panel shows head-jerk responding, the Phase 1 CR.
Head-jerk responding during the pre-CS period was very low throughout aversive conditioning, averaging only $0.6 \%, 1.3 \%, 0.0 \%$, and $0.0 \%$ for Groups $\mathrm{I} 1, \mathrm{I} 28, \mathrm{Nl}$, and N28, respectively, over the phase. The "high" score of $1.3 \%$ represents only 2 head-jerk observations out of the 160 total taken per group during the phase.

Freezing. The left portion of the upper panel of Figure 2 shows the acquisition of freezing during Phase 2 . The figure suggests that the interference groups were slower to acquire freezing to the CS than were the noninterference groups. The impression was confirmed by an interference $\times$ retention interval $\times$ trial ANOVA, which revealed significant effects of interference $[F(1,28)=$ $19.61]$ and trial $[F(3,84)=19.71]$, and an interference $\times$ trial interaction $[F(3,84)=3.96]$. No main effects or interactions involving retention interval were reliable $(F \mathrm{~s}<1)$. Planned comparisons confirmed that, overall, each interference group froze less during Phase 2 than did either of the noninterference groups [all $F \mathrm{~s}(1,28)>$ 8.99]. Under the present conditions, initial appetitive conditioning appeared to interfere with the subsequent acquisition of aversive responding.

Pre-CS freezing during Phase 2 averaged $0.0 \%, 5.6 \%$, $0.6 \%$, and $6.3 \%$ for Groups $\mathrm{I} 1, \mathrm{~N} 1, \mathrm{I} 28$, and $\mathrm{N} 28$, respectively. An interference $\times$ retention interval $\times$ trial ANOVA revealed main effects of interference $[F(1,28)=$ 8.52] and trial $[F(3,84)=8.16]$, and an interference $\times$ trial interaction $[F(3,84)=6.21]$. Prior appetitive conditioning in the interference groups appeared to reduce their acquisition of pre-CS freezing. No effects or interactions involving retention interval were reliable $(F s<1)$.

\section{Retention Test}

Head-jerk responding. The right-hand portion of Figure 2 suggests a strong recovery of head jerking at the 28-day retention interval (Group I28). The pattern was confirmed by an interference $\times$ retention interval $\times$ trial ANOVA on the head-jerk responding during the CS, which revealed significant effects of interference $[F(1,28)=43.70]$ and retention interval $[F(1,28)=$ $42.11]$, and an interference $\times$ retention interval interaction $[F(1,28)=53.85]$. No effects involving the trial factor were reliable $(F \mathrm{~s}<1)$. Planned comparisons revealed that Group 128 responded more than Group I1 $[F(1,28)=97.29]$; there was no difference between the corresponding noninterference groups $[F(1,28)<1]$. Head-jerk responding to the CS clearly recovered over time in the present interference group. Pre-CS head-jerk responding averaged only $0.4 \%, 0.4 \%, 0.4 \%$, and $0 \%$ for Groups I1, I28, N1, and N28 during the session. An average of $0.4 \%$ represents only one head-jerk observation out of a total of 240 pre-CS observations per group.

Freezing. The upper panel of Figure 2 suggests a complementary effect of retention interval on Phase 2 freezing. An interference $\times$ retention interval $\times$ trial ANOVA on CS freezing yielded effects of interference $[F(1,28)=$ $26.71]$ and retention interval $[F(1,28)=17.29]$, and an interference $\times$ retention interval interaction $[F(1,28)=$ 14.20]. There was a near-significant main effect of trial 
$[F(5,140)=2.16, p=.062]$, but trial did not interact with any other factor $[F \mathrm{~s}(5,140)<1.37]$. Planned comparisons indicated that Group I 28 froze significantly less than Group I1 $[F(1,28)=39.94]$; the difference between Groups N28 and N1 was not reliable $[F(1,28)<1]$. The analysis of pre-CS freezing revealed no corresponding differences in pre-CS scores $[F \mathrm{~s}(1,28)<2.21]$. Freezing during the pre-CS period averaged $11.2 \%, 10.0 \%$, $11.2 \%$, and $18.7 \%$ on the test for Groups $I 1, \mathrm{~N} 1, \mathrm{I28}$, and $\mathrm{N} 28$, respectively.

\section{Discussion}

The results of this experiment parallel those obtained in aversive-to-appetitive transfer (Experiment 1). At the 28-day interval, the Phase 1 behavior (head-jerk responding) had recovered, while the Phase 2 behavior (freezing) had become resuppressed. As before, this pattern suggests that Phase 2 can suppress Phase 1 responding without necessarily damaging what was learned in Phase 1. It also suggests that Phase 1's interference with Phase 2 performance can come about through a mechanism other than interference with learning. The fact that a similar general pattern was obtained in both appetitiveto-aversive and aversive-to-appetitive transfer suggests that it does not depend on an asymmetry between aversive and appetitive conditioning. At least with the methods used here, similar recovery effects were observed whether the aversive US was used in Phase 1 or in Phase 2 .

Initial appetitive conditioning interfered significantly with the acquisition of freezing during Phase 2 of this experiment. Although this result sits well with most views of cross-motivational transfer, it has not been obtained universally. In previous experiments, appetitive conditioning has interfered with subsequent aversive conditioning (Dickinson, 1976; Konorski \& Szwejkowska, 1956), but it has also facilitated it (DeVito \& Fowler, 1982; Scavio \& Gormezano, 1980) and had no effect on it as well (Jackson, 1974). In our own previous work with the present method, appetitive conditioning had no effect on subsequent aversive conditioning when the interference group was compared with a control that was analogous, but not identical, to the present noninterference controls (Peck \& Bouton, 1990, Experiment 3).

\section{GENERAL DISCUSSION}

In both aversive-to-appetitive and appetitive-to-aversive transfer, Phase 1 performance recovered and Phase 2 performance became resuppressed when groups were tested 28 days following the end of Phase 2 . These findings are consistent with what is known about the effects of retention interval in other interference paradigms. Retention intervals introduced following Phase 2 cause spontaneous recovery of Phase 1 performance after extinction; they can also cause recovery of Phase 1 performance in human verbal interference (see, e.g., Postman, Stark, \& Fraser, 1968; Underwood, 1948a, 1948b) and in animal discrimination reversal learning (see, e.g., Chiszar \&
Spear, 1969; Gleitman, 1971; Gordon \& Spear, 1973; Spear et al., 1980). The latter two paradigms may be especially consistent with the present data, because they also suggest that performance from Phase 2 is also resuppressed-that is, that proactive interference recovers over time, in similar settings. Put together, the results suggest that both proactive interference and retroactive interference may come about because interference occurs at retrieval or performance output rather than during learning.

This conclusion contrasts with a tradition in animal conditioning theory which often assumes that proactive and retroactive interference effects result from interference with what is learned during, or stored from, the target phase. As we noted in the introduction, the RescorlaWagner model and related models (see, e.g., Daly \& Daly, 1982) assume that Phase 2 damages, or causes the unlearning of, information stored in Phase 1. The same assumption can be found in many connectionist models of human learning and memory (see McCloskey \& Cohen, 1989). Current treatments of proactive interference make the related assumption that Phase 1 interferes with the subject's storage of information from the second phase. This view is clearly implied by the Rescorla-Wagner model's representation of reciprocal inhibition theory; it also dominates current thinking about other proactive interference paradigms such as latent inhibition, which is usually assumed to occur because the animal fails to attend to or process the CS during Phase 2 (see, e.g., Mackintosh, 1975; Pearce \& Hall, 1980; Wagner, 1978, 1981). We cannot rule out the possibility that there was some damage to Phase 1 storage or to Phase 2 learning in the present experiments. However, nothing in the data would require us to assume such damage. The present results clearly indicate that an emphasis on learning and storage deficits would be an oversimplification for the case of cross-motivational transfer.

Conditioning models of the last 20 years (see, e.g., Mackintosh, 1975; Pearce, 1987; Pearce \& Hall, 1980; Rescorla \& Wagner, 1972; Wagner, 1978, 1981) do not account for spontaneous recovery, partly because they have often assumed unlearning (but see Pearce, 1987; Pearce \& Hall, 1980; Wagner, 1981), and partly because they have never directly addressed the effects of long-term retention intervals. Skinner (1950) suggested that handling cues, or cues present during early parts of the session, might undergo little extinction (or by implication counterconditioning) during Phase 2; when reintroduced at the start of a spontaneous recovery test, they could cue Phase 1 performance. Since our groups tested at the 1and 28-day intervals received equivalent handling and early-session cues, this mechanism cannot account for the present results (see also Robbins, 1990; Thomas \& Sherman, 1986). More recently, Robbins (1990) suggested that attention to the CS, which might decline during extinction, might recover and make responding possible over the retention interval. It is not clear that this mechanism could be adapted easily to the present data. Over 28 days, 
responding shifted from a Phase 2 response to a response connected with Phase 1 . For an attentional mechanism to account for the shift, we would need to invoke responseor association-specific attentional processes, as well as a plausible reason for them to change over time.

An alternative approach could assume that information from both phases was adequately learned and stored in the present experiments. Spontaneous recovery effects could then result from the animal's forgetting which of the two phases occurred more recently (Gleitman, 1971; Mackintosh, 1974; Spear, 1971). Alternatively, performance in cross-motivational transfer (like that in other interference paradigms) could depend more directly on how well information from each phase is retrieved (see, e.g., Bouton, 1991).

Consider one way of building upon a retrieval account of extinction (see, e.g., Bouton, 1991). Instead of "unlearning" information from Phase 1 , the animal in a crossmotivational transfer experiment could acquire two new representations in Phase 2. First, as in extinction, it might learn a new T-no $\mathrm{US}_{1}$ relation (e.g., shock inhibition). Retrieval of this relation would interfere with $\mathrm{T}-\mathrm{US}_{1}$ in performance. Second, since the CS is also paired with a new US, the animal would also learn a new T-US ${ }_{2}$ relation, which would further interfere with $\mathrm{T}-\mathrm{US}_{1}$. In aversive-appetitive transfer, initial retrieval of $\mathrm{T}$-shock would interfere with retrieval of $\mathrm{T}$-food; but the learning and retrieval of $\mathrm{T}$-food and $\mathrm{T}$-no shock over Phase 2 would increasingly interfere with T-shock. A key element in the explanation of spontaneous recovery may be the further observation that inhibition (T-no shock) is forgotten more rapidly than excitation ( $\mathrm{T}$-shock) over time (Hendersen, 1978, 1985; Thomas, 1979). If retrieval of $\mathrm{T}$-no shock were to decrease over a retention interval, $\mathrm{T}$-food performance would decline, and $\mathrm{T}$-shock performance could recover. Both types of effect were demonstrated in the present experiments.

We may further suppose that the forgetting of inhibition is due to a failure to retrieve inhibition at the longer retention interval. Time may provide part of the background context in which all learning occurs; retrieval depends on the degree of match between the context present during testing with the context present during learning (see, e.g., Spear, 1978; Tulving, 1974). Forgetting over time may thus occur because of an increasing mismatch between the temporal testing and learning contexts. One advantage of this approach is that it would immediately integrate the effects of time (present results) with those of physical context (see, e.g., Peck \& Bouton, 1990): Spontaneous recovery can be viewed as the "renewal effect" that occurs when the animal is removed from the temporal Phase 2 context (Bouton, 1988; Bouton \& Swartzentruber, 1991). Given this sort of principle, it may be possible to integrate time, physical context, and interference effects as they occur in many of the Pavlovian interference paradigms.

Whether or not these particular mechanisms are accepted, the present results make clear that the retroactive and proactive interference effects in cross-motivational transfer can occur by some mechanism other than disruption of what is stored from, or learned during, the target phase. This conclusion is consistent with results from several other interference paradigms in Pavlovian learning (Bouton, 1991). It may be fruitful to view a variety of retroactive and proactive interference effects as problems in memory retrieval.

\section{REFERENCES}

Bolles, R. C., Collier, A. C. (1976). The effect of predictive cues on freezing in rats. Animal Learning \& Behavior, 4, 6-8.

Bouton, M. E. (1988). Context and ambiguity in the extinction of emotional learning: Implications for exposure therapy. Behaviour Research \& Therapy, 26, 137-149.

Bouton, M. E. (1991). Context and retrieval in extinction and in other examples of interference in simple associative learning. In L. Dachowski \& C. F. Flaherty (Eds.), Current topics in animal leaming: Brain, emotion, and cognition (pp. 25-53). Hillsdale, NJ: Erlbaum.

Bouton, M. E., \& Bolles, R. C. (1979). Contextual control of the extinction of conditioned fear. Learning \& Motivation, 10, 445-466.

Bouton, M. E., \& Bolles, R. C. (1980). Conditioned fear assessed by freezing and by the suppression of three different baselines. Animal Leaming \& Behavior, 8, 429-434.

Bouton, M. E., KING, D. A. (1983). Contextual control of the extinction of conditioned fear: Tests for the associative value of the context. Joumal of Experimental Psychology: Animal Behavior Processes, 9, 248-265.

Bouton, M. E., \& Peck, C. A. (1989). Context effects on conditioning, extinction, and reinstatement in an appetitive conditioning preparation. Animal Leaming \& Behavior, 17, 188-198.

Bouton, M. E., \&wartzentruber, D. (1991). Sources of relapse after extinction in Pavlovian and instrumental learning. Clinical Psychology Review, 11, 123-140.

BromaGe, B. K., \& SCAvio, M. J., JR. (1978). Effects of an aversive $\mathrm{CS}+$ and CS - under deprivation upon successive classical appetitive and aversive conditioning. Animal Leaning \& Behavior, 6, 57-65.

Chiszar, D. A., \& Spar, N. E. (1969). Stimulus change, reversal learning, and retention in the rat. Journal of Comparative \& Physiological Psychology, 69, 190-195.

DALY, H. B., \& DALY, J. T. (1982). A mathematical model of reward and aversive nonreward: Its application in over 30 appetitive learning situations. Journal of Experimental Psychology: General, 111, 441-480.

DAlY, H. B., DALY, J. T. (1987). A computer simulation/mathematical model of learning: Extension of DMOD from appetitive to aversive situations. Behavior Research Methods, Instruments, \& Computers, 19, 108-112.

DEVIro, P. L., \& Fowler, H. (1982). Transfer of conditioned appetitive stimuli to conditioned aversive excitatory and inhibitory stimuli. Learning \& Motivation, 13, 135-154.

Dickinson, A. (1976). Appetitive-aversion interactions: Facilitation of aversive conditioning by prior appetitive training in the rat. Animal Learning \& Behavior, 4, 416-420.

Dickinson, A., DeARING, M. F. (1979). Appetitive-aversive interactions and inhibitory processes. In A. Dickinson \& R. A. Boakes (Eds.), Mechanisms of leaming and motivation: A memorial volume to Jerzy Konorski (pp. 203-231). Hillsdale, NJ: Erlbaum.

FANSELow, M. S., \& Bolles, R. C. (1979). Naloxone and shock-elicited freezing in the rat. Journal of Comparative \& Physiological Psychology, 93, 736-744.

Gleitman, H. (1971). Forgetting of long-term memories in animals In W. K. Honig \& P. H. R. James (Eds.), Animal memory (pp. 144). New York: Academic Press.

Gluck, M., BowER, G. H. (1988). From conditioning to category learning: An adaptive network model. Joumal of Experimental Psychology: General, 117, 227-247. 
Gordon, W. C., \& SPEAR, N. E. (1973). Effect of reactivation of a previously acquired memory on the interaction between memories in the rat. Journal of Experimental Psychology, 99, 349-355.

HENDERSEN, R. W. (1978). Forgetting of conditioned fear inhibition. Learning \& Motivation, 9, 16-30.

Hendersen, R. W. (1985). Fearful memories: The motivational significance of forgetting. In F. R. Brush \& J. B. Overmier (Eds.), Affect, conditioning, and cognition: Essays on the determinants of behavior (pp. 43-54). Hillsdale, NJ: Erlbaum.

Holland, P. C. (1977). Conditioned stimulus as a determinant of the form of the Pavlovian conditioned response. Journal of Experimental Psychology: Animal Behavior Processes, 3, 77-104.

Holland, P. C. (1979). The effects of qualitative and quantitative variation in the US on individual components of Pavlovian appetitive conditioned behavior in rats. Animal Learning \& Behavior, 7, 424-432.

HowELL, D. C. (1987). Statistical methods for psychology. Boston: Duxbury.

JACKsON, D. E. (1974). CS-free food contingencies and subsequent acquisition of conditioned suppression: No transfer effect. Bulletin of the Psychonomic Society, 4, 235-236.

Konorski, J. (1967). Integrative activity of the brain. Chicago: University of Chicago Press.

KonORSKI, J., \& SzWEJKOWSKA, G. (1956). Reciprocal transformations of heterogeneous conditioned reflexes. Acta Biologiae Experimentalis, 17, 141-165.

Krank, M. D. (1985). Asymmetrical effects of Pavlovian excitatory and inhibitory aversive transfer on Pavlovian appetitive responding and acquisition. Learning \& Motivation, 16, 35-62.

MaCkintosh, N. J. (1974). The psychology of animal learning. London: Academic Press.

Mackintosh, N. J. (1975). A theory of attention: Variations in the associability of stimuli with reinforcement. Psychological Review, 82 , 276-298.

McCloskey, M., \& CoHEN, N. J. (1989). Catastrophic interference in connectionist networks: The sequential learning problem. In G. H. Bower (Ed.), The psychology of learning and motivation (Vol. 24, pp. 109-164). New York: Academic Press, Inc.

Pavlov, I. P. (1927). Conditioned reflexes. London: Oxford University Press.

Pearce, J. M. (1987). A model for stimulus generalization in Pavlovian conditioning. Psychological Review, 94, 61-73.

Pearce, J. M., \& Hall, G. (1980). A model for Pavlovian learning: Variations in the effectiveness of conditioned but not of unconditioned stimuli. Psychological Review, 87, 532-552.

Peck, C. A., Bouton, M. E. (1990). Context and performance in aversive-to-appetitive and appetitive-to-aversive transfer. Leaming and Motivation, 21, 1-31.

Postman, L., Stark, K., Frașer, J. (1968). Temporal changes in interference. Journal of Verbal Learning \& Verbal Behavior, 7 , 672-694.

Rescorla, R. A., \& Solomon, R. L. (1967). Two-process learning theory: Relationships between Pavlovian conditioning and instrumental learning. Psychological Review, 74, 151-182.
Rescorla, R. A., \& WAgner, A. R. (1972). A theory of Pavlovian conditioning: Variations in the effectiveness of reinforcement and nonreinforcement. In A. H. Black \& W. F. Prokasy (Eds.), Classical conditioning II: Current research and theory (pp. 64-99). New York: Appleton-Century-Crofts.

RobBins, S. J. (1990). Mechanisms underlying spontaneous recovery in autoshaping. Joumal of Experimental Psychology: Animal Behavior Processes, 16, 235-249.

SCAvio, M. J., JR. (1974). Classical-classical transfer: Effects of prior aversive conditioning upon appetitive conditioning in rabbits. Journal of Comparative \& Physiological Psychology, 86, 107-115.

SCAvio, M. J., JR. * Gormezano, I. (1980). Classical-classical transfer: Effects of prior appetitive conditioning upon aversive conditioning in rabbits. Animal Learning \& Behavior, 8, 218-224.

Shanks, D. R. (1991). Categorization by a connectionist network. Journal of Experimental Psychology: Learning, Memory, \& Cognition, $17,433-443$.

Skinner, B. F. (1950). Are theories of learning necessary? Psycho logical Review, 57, 193-216.

SPEAR, N. E. (1971). Forgetting as retrieval failure. In W. K. Honig \& P. H. R. James (Eds.), Animal memory (pp. 45-109). New York: Academic Press.

SPEAR, N. E. (1978). The processing of memories: Forgetting and retention. Hillsdale, NJ: Erlbaum.

SPEAR, N. E. (1981). Extending the domain of memory retrieval. In N. E. Spear \& R. R. Miller (Eds.), Information processing in animals: Memory mechanisms (pp. 341-378). Hillsdale, NJ: Erlbaum.

Spear, N. E., Smith, G. J., Bryan, R. G., Gordon, W. C., Timmons, R., Chiszar, D. A. (1980). Contextual influences on the interaction between conflicting memories in the rat. Animal Learning \& Behavior, 8, 273-281.

Tномаs, D. A. (1979). Retention of conditioned inhibition in a barpress suppression paradigm. Learning \& Motivation, 10, 161-177.

Thomas, D. R., \&herman, L. (1986). An assessment of the role of handling cues in spontaneous recovery after extinction. Journal of the Experimental Analysis of Behavior, 46, 305-314.

Tulving, E. (1974). Cue-dependent forgetting. American Scientist, 62 , 74-82.

UNDERWOOD, B. J. (1948a). Retroactive and proactive inhibition after five and forty-eight hours. Journal of Experimental Psychology, 38, 29-38.

UNDERWOOD, B. J. (1948b). "Spontaneous" recovery and verbal associations. Joumal of Experimental Psychology, 38, 429-439.

WAGNER, A. R. (1978). Expectancies and the priming of STM. In S. H. Hulse, H. Fowler, \& W. K. Honig (Eds.), Cognitive processes in animal behavior (pp. 177-209). Hillsdale, NJ: Erlbaum.

WAGNER, A. R. (1981). SOP: A model of automatic memory processing in animal behavior. In N. E. Spear \& R. R. Miller (Eds.), Information processing in animals: Memory mechanisms (pp. 5-47). Hillsdale, NJ: Erlbaum.

(Manuscript received February 14, 1992; revision accepted for publication May 27, 1992.) 